\title{
Influence of Pump Noise on Mode-locked Fiber Oscillators
}

\author{
Tesfay G. Teamir ${ }^{1}$, Parviz Elahi ${ }^{1}$, I. Levent Budunoglu ${ }^{1}$, Kutan Gürel ${ }^{1}$, and F. Ömer \\ Ilday $^{1,2}$ \\ ${ }^{1}$ Department of Physics, Bilkent University, 06800 Ankara,, Turkey \\ ${ }^{2}$ Department of Electrical and Electronics Engineering, Bilkent University, 06800 Ankara,, Turkey \\ tesfay.teamir@bilkent.edu.tr
}

\begin{abstract}
Pump modulation transfer function (MTF), and its dependence on pump power are investigated for all normal dispersion, dispersion managed and soliton-like mode-locked oscillator both in experiment and simulation. We find that cavity losses and pulse instabilities such as multiple pulsing influence noise transfer, strongly.
\end{abstract}

OCIS codes: (140.3510) Lasers, fiber; (140.3615) Lasers, ytterbium; (140.7090) Ultrafast lasers; (190.0190 ) Nonlinear optics..

\section{Introduction}

Intensity noise below resonant relaxation oscillation frequency of lasers is dominated by pump noise that can resulted from technical, mechanical, thermal, electrical fluctuations, or intentionally introduced pump modulation. Noise level in this frequency range has been used to characterize intensity noise of laser systems. Different theoretical models and experiments are used to predict signal intensity noise which is transferred from pump intensity fluctuations. In this paper we report a systematic study of transfer of Intensity modulation of pump to the output signal (MTF) for different regimes of mode-locked oscillators, all-normal-dispersion, dispersion-managed, and soliton-like both on theory and experiment.

\section{Results}

\subsection{Theoretical Model}

Here we used a theoretical model for calculation of transfer of pump modulation to output signal of a mode-locked oscillator. In this model, we considered fiber oscillator as an amplifier and a resonator with a nonlinear transmission feedback. Transfer of pump and signal fluctuations to the output of amplifier is modeled as in Ref. [1] and can be described by

$$
q_{s(n)}^{\prime}(z, \omega)=\xi_{s(n)}(\omega) q_{s(n)}(z, \omega)+\xi_{p(n)}(\omega) q_{p(n)}(z, \omega)
$$

where $q(z, \omega)$ represent the individual modulation amplitude at modulation frequency $\omega . s$ and $p$ stand for signal and pump, respectively, and $n$ denotes number of roundtrips in oscillator. $q$ is modulation on amplitude signal output from amplifier which some portion of it re-feed into the amplifier as a seed. By introducing a nonlinear transmittance, $T\left(P_{s(n)}^{\prime}\right)$, the transmitted modulation amplitude, $q^{\prime \prime}(z, \omega)$, will be

$$
q_{s(n)}^{\prime \prime}(z, \omega)=\left[\xi_{s(n)}(\omega) q_{s(n)}(z, \omega)+\xi_{p(n)}(\omega) q_{p(n)}(z, \omega)\right]-\beta\left[\xi_{s(n)}(\omega) q_{s(n)(z, \omega)}+\xi_{p(n)}(\omega) q_{p(n)}(z, \omega)\right]^{2}
$$

where $\beta$ is a constant parameter. By introducing $a=-\beta\left|\xi_{s(n)}\right|^{2} e^{2 i \phi_{s(n)}}, b=\left|\xi_{s(n)}\right| e^{i \phi_{s(n)}}\left(1-2 \beta q_{p(n)}\left|\xi_{p(n)}\right| e^{i \phi_{p(n)}}\right)$ and $c=q_{p(n)}\left|\xi_{p(n)}\right| e^{i \phi_{p(n)}}\left(1-\beta q_{p(n)}\left|\xi_{p(n)}\right| e^{i \phi_{p(n)}}\right)$, the governed equation for modulation amplitude of signal yields

$$
q_{s(n+1)}=a q_{s(n)}^{2}+b q_{s(n)}+c
$$

Finally, the pump modulation transfer to output signal of the oscillator is given by $\xi_{p}(\omega)=\left|\frac{q_{s(n+1)}(z, \omega)}{q_{p}}\right|, n \longrightarrow \infty$ 

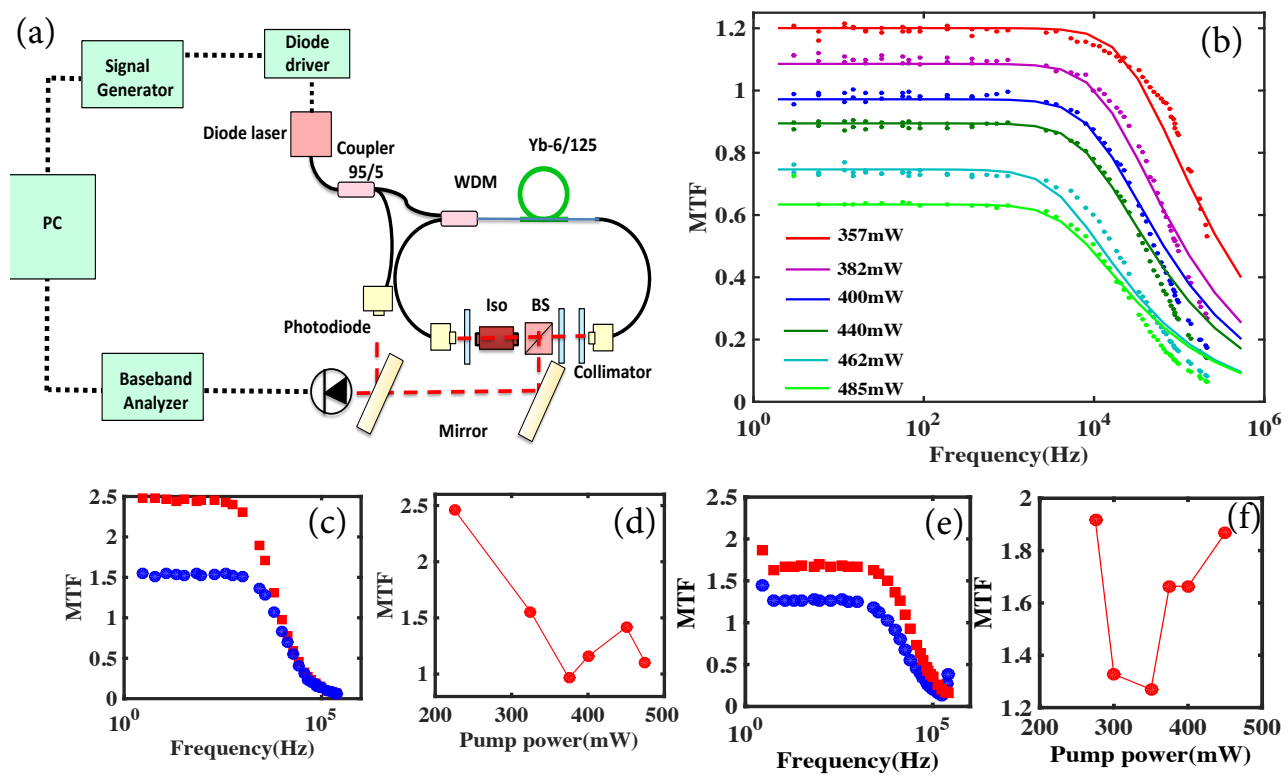

Fig. 1. (a) Scematic setup for the intensity noise measurement. (a) Measured (circles) and calculated (solid line) for modulation transfer function (MTF) for pump in ANDi oscillator for different pump power. (c) Measured MTF for $225 \mathrm{~mW}$ (squares) and $325 \mathrm{~mW}$ (circles) pump power in dispersionmanaged oscillator. (d) MTF vs pump power at $20 \mathrm{~Hz}$ pump modulation in the same oscillator. (e) Measured MTF for $275 \mathrm{~mW}$ (squares) and 350 $\mathrm{mW}$ (circles) pump power in solitonlike oscillator. (f) MTF vs pump power at $20 \mathrm{~Hz}$ pump modulation in the same oscillator.

\subsection{Experimental Results}

The pump modulation transfer to signal (MTF) characterization is done using the standard method described in Ref. [2]. The pump power is modulated by modulating the diode's current over the frequency range of $1 \mathrm{~Hz}-250 \mathrm{kHz}$. The MTF measured via the same method described in Ref. [2]. The MTF was measured for different mode-locked oscillators, all normal dispersion (ANDi), dispersion-managed, and solitonlike oscillator. Fig. 1(a) shows the schematic characterization setup for ANDi oscillator. The measured and calculated MTF for different diode pump power is shown in Fig. 1(b). At frequencies lower than cutoff frequency, MTF decreases linearly by increasing pump power. The measured MTF dispersion-managed oscillator is shown in Fig. 1(c). As we see, MTF increases because of increasing of cavity losses due to implementing diffraction grating inside the oscillator. The measured MTF for different input pump power is shown in Fig. 1(d). An increasing of MTF by increasing pump power is observed between 370mW$430 \mathrm{~mW}$ which is due to onset of multiple pulsing. The same but more significant behavior is observed for soliton like oscillators(Fig. 1(f)) with highly negative total dispersion delay.

\section{Conclusion}

In conclusion, we report the modulation transfer function in a different mode-locked regimes both in theory and experiment with the following main results: (1) MTF increases by increasing cavity losses. (2) The pulse instability such as onset of multiple pulsing increases MTF and leads to a relative increases of noise transfer.

\section{References}

1. K. Gürel, P. Elahi, L. Budunoğlu, C. Şenel, P. Paltani, and F. Ö. Ilday, "Prediction of pulse-to-pulse intensity fluctuation characteristics of high power ultrafast fiber amplifiers," Appl. Phys. Lett. 105, 011111 (2014)

2. I. L. Budunoğlu, C. Ülgüdür, B. Oktem, and F. Ö. Ilday,“Intensity noise of mode-locked fiber lasers,” Opt. Lett. 34, 25161 (2009) 